Document downloaded from:

http://hdl.handle.net/10251/82415

This paper must be cited as:

Antoni-Alandes, R.; Dietrich, D.; Bennett, MJ.; Rodríguez Egea, PL. (2016). Hydrotropism: Analysis of the Root Response to a Moisture Gradient. Environmental Responses in Plants. 3-9. doi:10.1007/978-1-4939-3356-3_1.

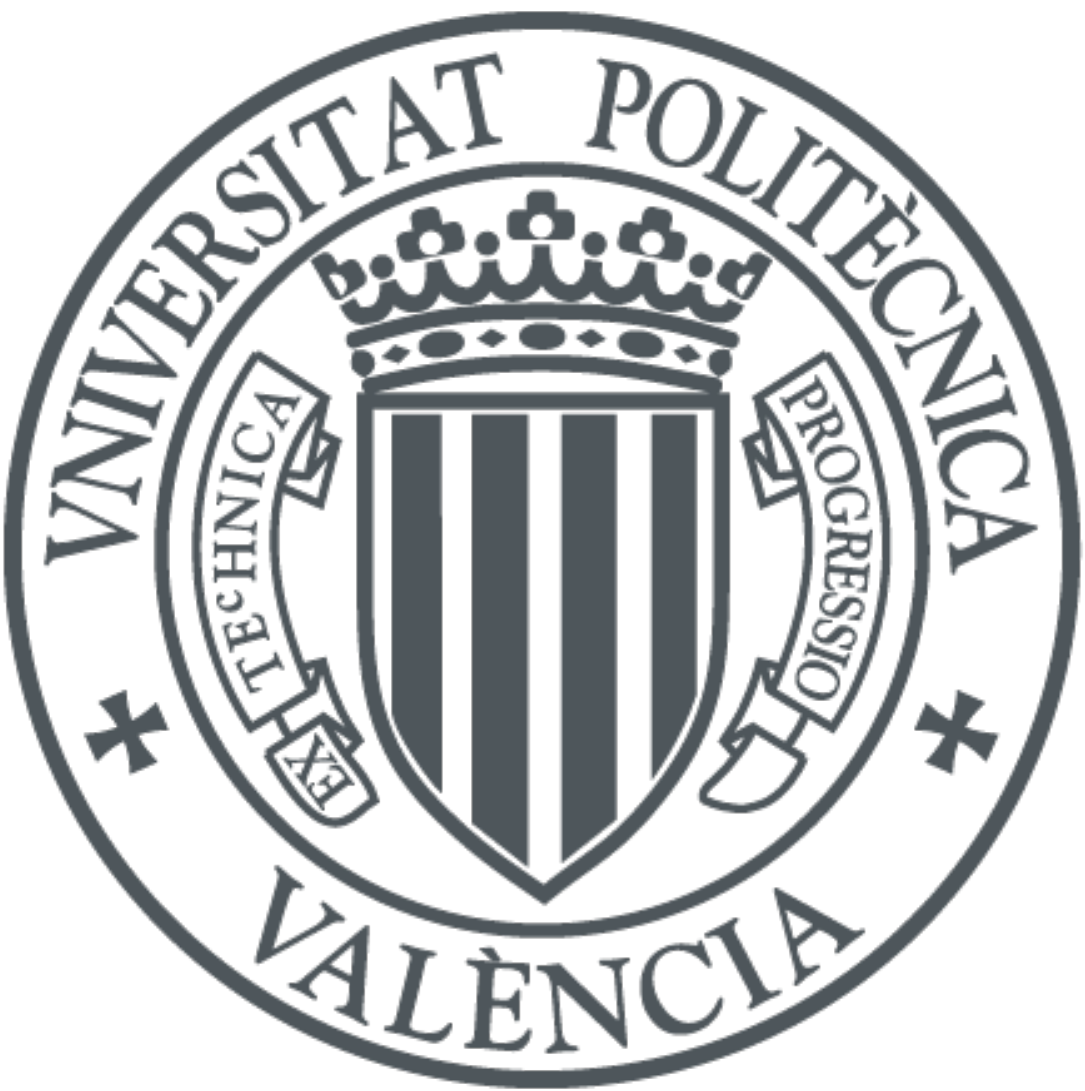

The final publication is available at

http://doi.org/10.1007/978-1-4939-3356-3_1

Copyright Springer New York

Additional Information 


\section{Hydrotropism: analysis of the root response to a moisture gradient}

Regina Antoni*, Daniela Dietrich*, Malcolm J. Bennett and Pedro L. Rodriguez†

Instituto de Biología Molecular y Celular de Plantas, Consejo Superior de Investigaciones Científicas-Universidad Politécnica de Valencia, 46022 Valencia, Spain (P.L.R.)

Centre for Plant Integrative Biology, University of Nottingham, LE12 5RD, UK (R.A., D.D., M.J.B.)

${ }^{*}$ Both authors contributed equally to this work

†To whom correspondence should be addressed;

e-mail prodriguez@ibmcp.upv.es; phone: 34963877860

Running title: Hydrotropic root response to a moisture gradient 


\section{Summary}

Hydrotropism is a genuine response of roots to a moisture gradient to avoid drought. An experimental system for the induction of hydrotropic root response in Petri dishes was designed by pioneering groups in the field. This system uses split agar plates containing an osmolyte only in a region of the plate in order to generate a water potential gradient. Arabidopsis seedlings are placed on the MS agar plate so that their root tips are near the junction between plain MS medium and the region supplemented with the osmolyte. It elicits a hydrotropic response in Arabidopsis roots that can be measured as the root curvature angle.

Keywords: hydrotropism, water potential gradient, root curvature angle, moisture gradient, root growth, sorbitol, ABA, Arabidopsis

\section{Introduction}

Drought is a major environmental stress that affects plant growth and has a serious impact on agriculture. Plants have developed a range of mechanisms to overcome drought stress including stomatal closure, regulation of gene expression, accumulation of osmoprotectants or compatible solutes and modulation of growth (1). Hydrotropism forms part of the droughtavoidance plant response and is a mechanism by which roots change growth direction according to differences in the water potential of the soil. Elucidating the molecular mechanism of hydrotropism in roots is important both for understanding plant adaptation to soil moisture gradients and improving crop productivity. Root growth is strongly directed by gravity and also by other tropisms like photo-, thigmo- or hydrotropism (2). The study of this latter process is complicated by the fact that gravitropism and hydrotropism influence each other and make it difficult to observe hydrotropism in isolation. However, since roots of the pea mutant ageotropum are agravitropic but hydrotropic both sensing pathways must operate through independent mechanisms (3). Additionally, the non-hydrotropic Arabidopsis mutants no hydrotropic response (nhr1) and mizu-kussei (miz1) show a normal root 
gravitropic response $(4,5)$. Unlike gravitropism, where the central role of auxin in its regulation has been well described, the molecular mechanisms that regulate hydrotropism are still largely unknown. Screening procedures in model plant species are needed for genetic dissection of complex biological processes. Therefore, a protocol that allows the analysis of the hydrotropic response in Arabidopsis seedlings can help to isolate key genes involved in this response. Takahashi et al. (6) and Eapen et al. (4) described two methods to measure the hydrotropic response in Arabidopsis seedlings. Both methods are based on the same principle of challenging roots with a water potential gradient that will ultimately trigger root bending. Here we describe one of them based on the use of split agar plates that contain two media with different water potential. The use of these strategies has allowed the isolation of mutants that lack a normal hydrotropic response such as miz1, miz2/gnom, ahr1 and $n h r 1(6-8,4)$, or the identification of mutants that show an enhanced hydrotropic response, such as the pp2c quadruple mutant impaired in the protein phosphatases type $2 \mathrm{C}$ that negative regulate ABA signalling (9). As a result, it has been identified a gene, MIZ1, that plays an essential role for hydrotropism and is expressed in the columella cells of the root cap (6). It has been also found that GNOM-mediated vesicular trafficking and ABA signalling through PYR/PYL/RCAR receptors is required for hydrotropism in Arabidopsis (7, 9). Finally, in addition to ABA $(5,9)$, cytokinins also play a role in root hydrotropism (8).

\section{Materials:}

- D-Sorbitol

- Murashige and Skoog (MS) basal salt macronutrient solution (10)

- $120 \times 120 \times 17$ mm square polystyrene Petri dishes

- 3M Micropore surgical tape

- Growth chamber in darkness

- Imaging system (optional, if time course analysis is intended): IR camera system (11)

- NIH image software ImageJ v1.37 


\section{Methods:}

\subsection{Growth of Arabidopsis seedlings}

1- Sterilise Arabidopsis seeds by treatment with $50 \%$ sodium hypochlorite for 5 minutes followed by four washes with sterile distilled water and stratify them in the dark at $4^{\circ} \mathrm{C}$ for 3 days.

2- Sow the seeds on plates containing half-strength (0.5x) MS medium, $\mathrm{pH}$ 5.6-5.8 and solidified with $1 \%$ agar (0.5xMS plates).

3- Seal the plates with $3 \mathrm{M}$ Micropore surgical tape and put them vertically in a growth chamber with $24 \mathrm{~h}$ light at $22^{\circ} \mathrm{C}$ for $5-6$ days.

\subsection{Preparation of plates that establish a water potential gradient}

1- The osmotic pressure ( $\pi$ ) of an ideal solution can be approximated using the Morse equation $\pi=$ iMRT, where $M$ is the molarity of the solute. Sorbitol is a common solute in laboratory that lacks secondary effects and we use it to generate a water potential gradient in split agar plates. For instance, adding $0.4 \mathrm{M}$ sorbitol as solute lowers the water potential by $-1 \mathrm{MPa}$. Split agar plates are generated by filling the upper region of the square Petri dish with medium containing $0.5 \times \mathrm{MS}$ salts and the lower region with $0.5 \times \mathrm{MS}$ salts $+0.4 \mathrm{M}$ sorbitol (Fig. 1). Thus, a water potential gradient will be generated between the two different media. Root tips will be located at the border between both media and the root curvature will be measured within 12 h (Fig. 1). The water potential gradient between both media becomes smaller over time, but exists for a substantial portion of the experimental period.

2- The split agar plates must be prepared just before the experiment. We use a template placed below the plate in order to guide the diagonal cutting of the medium with a scalpel (Fig. 1). Next, we remove the 0.5xMS medium from the lower part of 
the dish and we replenish the empty part of the plate with $0.5 \times \mathrm{MS}$ medium supplemented with $0.4 \mathrm{M}$ sorbitol.

3- Use the same template to guide the transfer of Arabidopsis seedlings grown on vertical plates to the plates with a water potential gradient so that their root tips are 23 millimetres above the border between the two media (blue line in Fig. 1). Seedlings should be transferred only to the 3 central rectangles. According to the size of the square Petri dishes, 5-6 seedlings of each genotype can be transferred to one of the rectangles in such a way that each plate contains up to 3 different genotypes.

4- At least three plates per experiment are needed. In order to avoid a positional effect, the order of the genotypes on the plates should be varied. The two rectangles close to the edges of the plate should be left empty as diffusion of water and sorbitol could be affected by boundary effects.

5- Seal the plates with $3 \mathrm{M}$ Micropore surgical tape to allow gas exchange.

\subsection{Root curvature measurement}

1- Place the plates vertically in a growth chamber at $22^{\circ} \mathrm{C}$ in darkness and take images of the plants after 8-13 h. Performing the experiment in darkness eliminates the effect of phototropism on root angle, although it has been described that the hydrotropic curvature of light-grown seedlings is notably higher than in dark-grown seedlings (12). In wild type plants root curvature generated in response to the moisture gradient can be observed from the first hour onwards; however, after 8-13 $\mathrm{h}$ the angle values of root curvature will be higher, which will make it easier to distinguish between altered and normal hydrotropic response (Fig. 2A, B). If detailed analysis of the development of the hydrotropic root curvature over time is intended, images should be taken with an automated IR camera system.

2- Use Image $\mathrm{J}$ to measure the curvature angle of the primary root (Fig. 2A). 


\section{Notes:}

1- After the preparation of the plates, the water potential gradient will decrease over time (6). For this reason, plates must be prepared just before the start of the experiment and angle measurements should be taken after 8-13 $\mathrm{h}$.

2- While setting up the experiment, maintain plants in vertical orientation as much as possible. As roots tend to grow in the direction of gravity, keeping them in the same initial position will avoid unnecessary perturbations in root growth.

3- When preparing the plates take special care of working on a level surface to ensure that the level between the two media is similar. We use a spirit level to ensure the flow laminar hood where media are poured is really on the same level. Additionally take care to obtain a horizontal surface in the border between the two media that generate the water potential gradient. The transition between both media should be as flat as possible since irregular surfaces between both media could alter the root hydrotropic response.

4- It is necessary to include in the experiment control $0.5 x M S$ plates lacking the water potential gradient. As shown in Fig. 2C, seedlings growing on control plates follow the gravity vector while those growing on plates that contain a water potential gradient bend and avoid the low water potential area. Once the measurement of root curvature is done, seedlings can be left growing for 2-3 days to observe if the response persists in time (Fig. 2D).

5- As temperature affects osmolyte diffusion and in order to keep the water potential gradient as steep as possible, cool down the $0.5 \mathrm{xMS}$ medium supplemented with sorbitol as much as possible before pouring.

6- Select seedlings of the same developmental stage, which should have similar root growth rates. Under our growth conditions $\left(22^{\circ} \mathrm{C}\right.$ in darkness) Col-0 plants show a root curvature of 30-40 degrees after $12 \mathrm{~h}$. In addition to the wild type control, the use 
of mutants that lack or show enhanced hydrotropic response is strongly advisable

(Fig. 2D).

\section{Acknowledgment}

Funding was provided by a Marie Curie Intra-European Fellowship to R.A and grants from the Ministerio de Ciencia e Innovacion, Fondo Europeo de Desarrollo Regional and Consejo Superior de Investigaciones Cientificas to P.L.R. (BIO2011-23446) and the Biotechnology and Biological Sciences Research Council to D.D and M.J.B.

\section{References}

1. Verslues,P.E., Agarwal,M., Katiyar-Agarwal,S., et al. (2006). Methods and concepts in quantifying resistance to drought, salt and freezing, abiotic stresses that affect plant water status. Plant J. 45, 523-539.

2. Roy, R. and Bassham, D. C. (2014) Root growth movements: Waving and skewing. Plant Science 221, 42-47.

3. Jaffe, M.J., Takahashi,H. and Biro,R.L. (1985) A pea mutant for the study of hydrotropism in roots. Science 230, 445-447.

4. Eapen, D., Barroso, M.L., Campos, M.E., et al. (2003) A no hydrotropic response (nhr1) root mutant that responds positively to gravitropism in Arabidopsis. Plant Physiology 131, 536-546.

5. Kobayashi, A., Takahashi, A., Kakimoto, Y., et al. (2007) A gene essential for hydrotropism in roots. Proc Natl Acad Sci USA 104, 4724-4729.

6. Takahashi, N., Goto, N., Okada, K. et al. (2002) Hydrotropism in abscisic acid, wavy, and gravitropic mutants of Arabidopsis thaliana. Planta 216, 203-211.

7. Miyazawa, Y., Takahashi, A., Kobayashi, A., et al. (2009) GNOM-mediated vesicular trafficking plays an essential role in hydrotropism of Arabidopsis roots. Plant Physiol 149, 835-840.

8. Saucedo, M., Ponce, G., Campos, M., et al. (2012) An altered hydrotropic response (ahr1) mutant of Arabidopsis recovers root hydrotropism with cytokinin. Journal of Experimental Botany 63, 3587-3601.

9. Antoni, R., Gonzalez-Guzman, M., Rodriguez, L., et al. (2013). PYRABACTIN RESISTANCE1-LIKE8 plays an important role for the regulation of abscisic acid signaling in root. Plant Physiol 161, 931-941.

10. Murashige, T. \& Skoog, F. (1962) A Revised Medium for Rapid Growth and Bio Assays with Tobacco Tissue Cultures. Physiologia Plantarum 15, 473-497.

11. Wells, D. M., French, A. P., Naeem, A., et al. (2012) Recovering the dynamics of root growth and development using novel image acquisition and analysis methods Philosophical Transactions of the Royal Society B: Biological Sciences 367, 2245.

12. Moriwaki,T., Miyazawa,Y., Fujii,N. et al. (2012) Light and abscisic acid signalling are integrated by MIZ1 gene expression and regulate hydrotropic response in roots of Arabidopsis thaliana. Plant Cell Environ. 35, 1359-1368. 


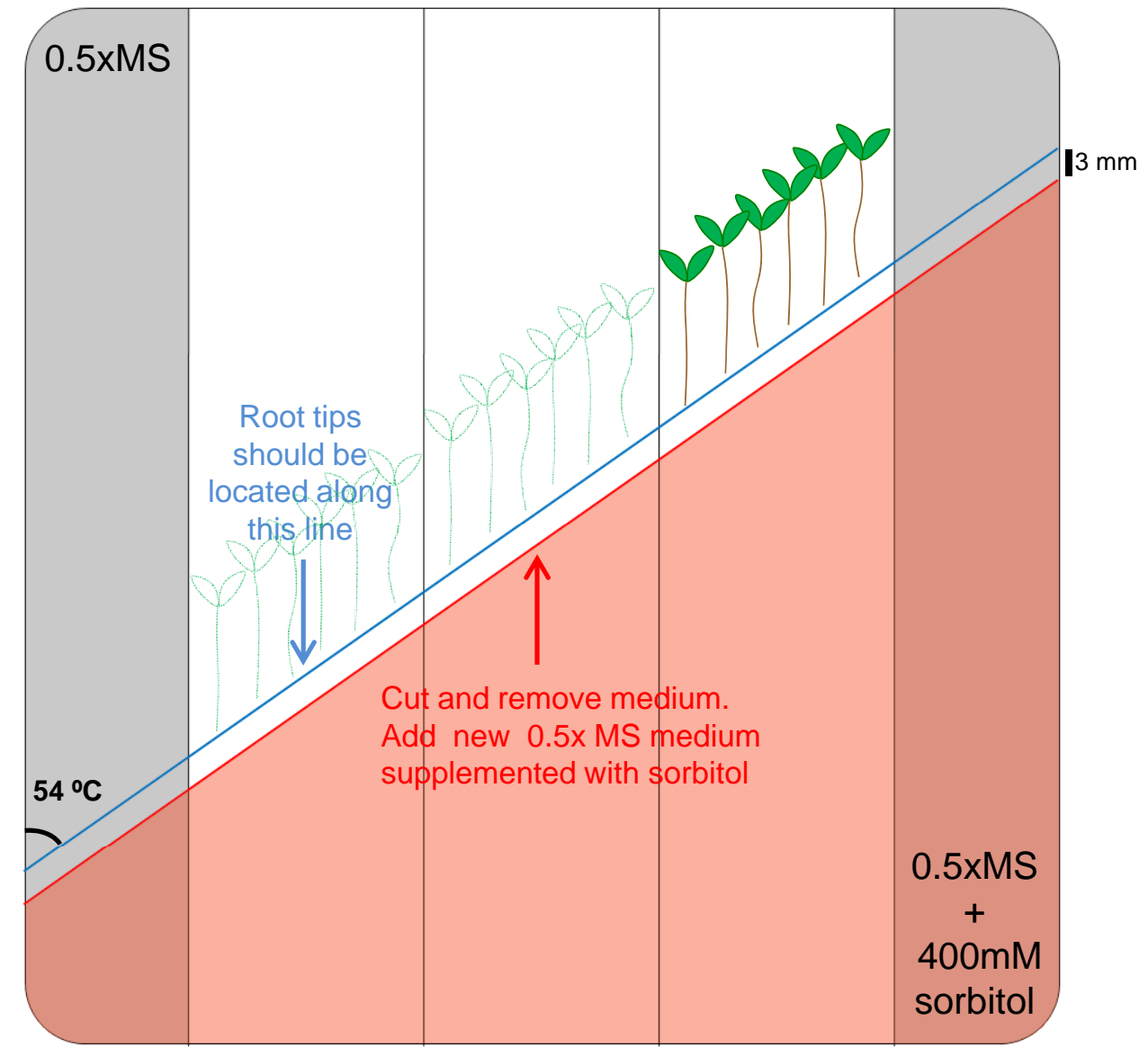

Figure 1. Schematic representation of the split agar plate used for the analysis of the root hydrotropic response. The upper part of the plate contains $0.5 \times \mathrm{MS}$ medium while the lower part contains $0.5 \times \mathrm{MS}$ medium supplemented with $0.4 \mathrm{M}$ sorbitol, which generates a water potential gradient. The plate is vertically divided in 5 rectangles. Seedlings should be located in the 3 central rectangles. 


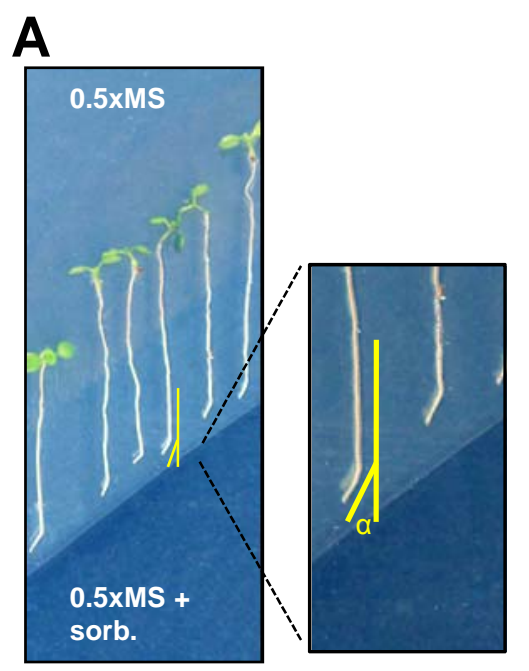

B
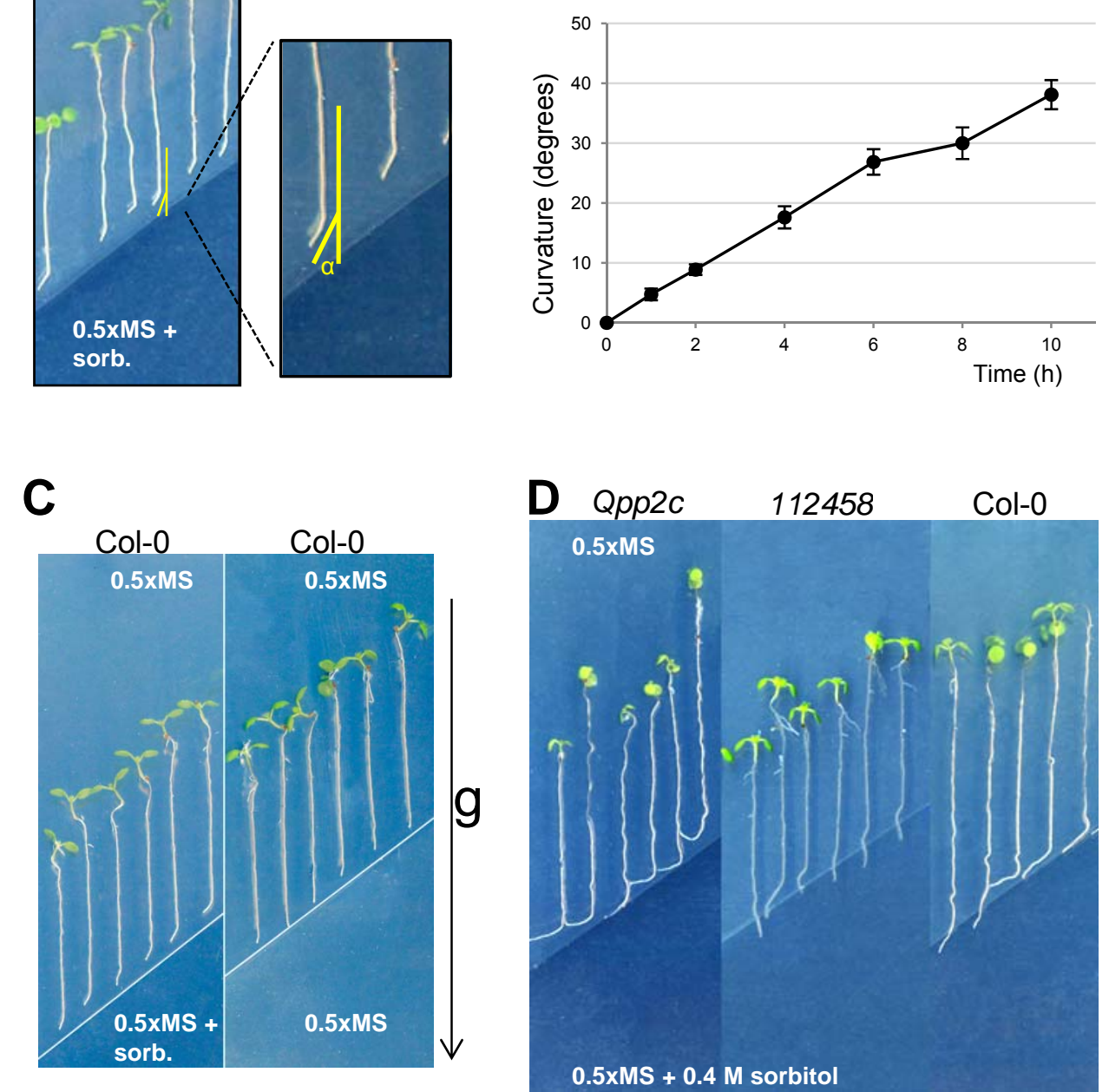

Figure 2. Root hydrotropic response of 6-day-old Arabidopsis seedlings in plates where a water potential gradient has been established. (A) Measurement of the root curvature angle $(\alpha)$ after $10 \mathrm{~h}$ of transferring the seedlings to split agar plates to estimate the hydrotropic response. (B) Time course of the hydrotropic response in Col-0 Arabidopsis seedlings. (C) Root bending only occurs in Col-0 seedlings after transferring them to plates with a water potential gradient. Otherwise, they follow the gravity (g) vector. (D) Root hydrotropic response of the ABA-hypersensitive mutant impaired in four clade $A$ PP2Cs (abi1-2 hab1-1 pp2ca-1 abi2-2, abbreviated as Qpp2c) and the ABA-insensitive mutant impaired in six PYR/PYL ABA receptors (pyr1 pyl1 pyl2 pyl4 pyl5 pyl8, abbreviated as112458). 6-day-old seedlings were transferred to plates with a moisture gradient and photographs were taken after 3 days. An enhanced hydrotropic response was observed in Qpp2c, whereas a lack of response was found in 112458 compared to Col-0 wt seedlings. 